\title{
Video Article \\ Application of a NMDA Receptor Conductance in Rat Midbrain Dopaminergic Neurons Using the Dynamic Clamp Technique
}

\author{
Collin J Lobb ${ }^{1}$, Carlos A Paladini ${ }^{1}$ \\ ${ }^{1}$ Neurosciences Institute, University of Texas San Antonio - UTSA \\ Correspondence to: Carlos A Paladini at carlos.paladini@utsa.edu \\ URL: https://www.jove.com/video/2275 \\ DOI: doi:10.3791/2275
}

Keywords: Neuroscience, Issue 46, electrophysiology, dynamic clamp, rat, dopamine, burst, RTXI

Date Published: $12 / 21 / 2010$

Citation: Lobb, C.J., Paladini, C.A. Application of a NMDA Receptor Conductance in Rat Midbrain Dopaminergic Neurons Using the Dynamic Clamp Technique. J. Vis. Exp. (46), e2275, doi:10.3791/2275 (2010).

\section{Abstract}

Neuroscientists study the function of the brain by investigating how neurons in the brain communicate. Many investigators look at changes in the electrical activity of one or more neurons in response to an experimentally-controlled input. The electrical activity of neurons can be recorded in isolated brain slices using patch clamp techniques with glass micropipettes. Traditionally, experimenters can mimic neuronal input by direct injection of current through the pipette, electrical stimulation of the other cells or remaining axonal connections in the slice, or pharmacological manipulation by receptors located on the neuronal membrane of the recorded cell.

Direct current injection has the advantages of passing a predetermined current waveform with high temporal precision at the site of the recording (usually the soma). However, it does not change the resistance of the neuronal membrane as no ion channels are physically opened. Current injection usually employs rectangular pulses and thus does not model the kinetics of ion channels. Finally, current injection cannot mimic the chemical changes in the cell that occurs with the opening of ion channels.

Receptors can be physically activated by electrical or pharmacological stimulation. The experimenter has good temporal precision of receptor activation with electrical stimulation of the slice. However, there is limited spatial precision of receptor activation and the exact nature of what is activated upon stimulation is unknown. This latter problem can be partially alleviated by specific pharmacological agents. Unfortunately, the time course of activation of pharmacological agents is typically slow and the spatial precision of inputs onto the recorded cell is unknown.

The dynamic clamp technique allows an experimenter to change the current passed directly into the cell based on real-time feedback of the membrane potential of the cell (Robinson and Kawai 1993, Sharp et al., 1993a,b; for review, see Prinz et al. 2004). This allows an experimenter to mimic the electrical changes that occur at the site of the recording in response to activation of a receptor. Real-time changes in applied current are determined by a mathematical equation implemented in hardware.

We have recently used the dynamic clamp technique to investigate the generation of bursts of action potentials by phasic activation of NMDA receptors in dopaminergic neurons of the substantia nigra pars compacta (Deister et al., 2009; Lobb et al., 2010). In this video, we demonstrate the procedures needed to apply a NMDA receptor conductance into a dopaminergic neuron.

\section{Video Link}

The video component of this article can be found at https://www.jove.com/video/2275/

\section{Protocol}

\section{Slice Preparation}

1. Cut brain slices using a vibrating microtome. We prepared $240 \mu \mathrm{m}$ horizontal midbrain slices from an isoflurane-anethetized Sprague-Dawley rat (Charles River Laboratories) using a vibrating microtome (Microm HM 650V) in accordance with the University of Texas at San Antonio Institutional Animal Care and Use Committee.

2. Keep the slices in an incubation chamber until you are ready to record. We use an incubation container heated to $32^{\circ} \mathrm{C}$ and filled with artificial cerebrospinal fluid (aCSF; in mM): $126 \mathrm{NaCl}, 2.5 \mathrm{KCl}, 1.25 \mathrm{NaH}_{2} \mathrm{PO}_{4}, 4 \mathrm{MgCl}_{2}, 2 \mathrm{CaCl}_{2}, 10$ dextrose, $25 \mathrm{NaHCO}_{3}, 1.3$ ascorbic acid, 2.4 sodium pyruvate and 0.05 glutathione.

\section{Electrophysiological Recording}

1. Transfer the slice to the intracellular recording rig in which an artificial cerebrospinal fluid (aCSF) at $35^{\circ} \mathrm{C}$ is being perfused. We use the same aCSF as in 1.2 except that $2 \mathrm{mM} \mathrm{MgCl}_{2}$ was used and glutathione was omitted. For horizontally prepared slices, we typically bisect the slice along the midline. 
2. Visualize the target neuron. We visualized individual substantia nigra dopaminergic neurons with a gradient contrast imaging system.

3. Pull an electrode using an electronic electrode puller. We pull electrodes with a tip resistance of 4-10 M $\Omega$ using a P97 Micropipette puller (Sutter Instrument Company).

4. Fill an electrode with the desired internal solution. We use a solution containing (in $\mathrm{mM}$ ): $138 \mathrm{~K}$-gluconate, $10 \mathrm{HEPES} 2 \mathrm{MgCl} 2,0.2 \mathrm{EGTA}$, $0.0001 \mathrm{CaCl}_{2}, 4 \mathrm{Na}-\mathrm{ATP}, 0.4 \mathrm{Na}-\mathrm{GTP}$. The internal solution was adjusted to a pH of 7.3 using $1 \mathrm{M} \mathrm{KOH}$ and an osmolarity of $270-275 \mathrm{mOsms}$.

5. Make a gigaohm seal onto the desired neuron. Rupture the seal with suction. This constitutes a whole cell recording. A Multiclamp $700 \mathrm{~B}$ amplifier was used in our configuration. The amplifier should then be placed in the 'I=0' current clamp mode.

\section{Conductance Application with Dynamic Clamp}

1. RTXI (www.rtxi.org) was executed on the dynamic clamp computer. A custom written model containing an NMDA receptor was loaded into memory. The current to be injected into the cell in real-time is calculated by the following equation:

$\mathrm{I}_{\mathrm{NMDA}}=-\mathrm{g}_{\mathrm{NMDA}}{ }^{*}\left[1 /\left(1+([\mathrm{Mg}] / 3.57){ }^{*} \mathrm{e}\left(\mathrm{V}_{\mathrm{m}}{ }^{* 0.062}\right)\right)\right]$ * $\left(\mathrm{V}_{\mathrm{m}}-\mathrm{E}_{\mathrm{NMDA}}\right)$ where $\mathrm{g}_{\mathrm{NMDA}}$ is the desired conductance (in $\mathrm{nS}$; by default set to $\left.0 \mathrm{nS}\right)$, [Mg] is the magnesium concentration (set to $1.5 \mathrm{mM}$ in our example below), $\mathrm{E}_{\mathrm{NMDA}}$ is the reversal potential for the NMDA receptor (set to $0 \mathrm{mV}$ ), and $V_{m}$ is the membrane potential of the cell measured from the amplifier (in millivolts).

2. The output from the dynamic clamp computer was connected to the Command input of the amplifier via an analog-to-digital converter.

3. The amplifier was placed in the 'IC' current clamp mode.

4. Enter your desired NMDA receptor conductance into RTXI (e.g. 40nS). You should see a phasic burst of action potentials. Alternatively, a conductance can be given to RTXI via an analog output (Figure $\left.\left.1 \mathrm{~A}, \mathrm{~g}^{\mathrm{g}} \mathrm{g}(\mathrm{t})\right)^{\prime}\right)$. An appropriate scaling factor must be used within RTXI to convert the signal from volts to Siemens.

\section{Representative Results}

A successful setup for application of a conductance using the dynamic clamp is shown in Figure $1 \mathrm{~A}$. Using this setup, we made a whole cell somatic recording from a dopaminergic neuron in the substantia nigra pars compacta. Dopaminergic cells typically fire spontaneously at low rates with a pacemaker-like pattern. A burst of action potentials can be evoked by phasic application of a NMDA receptor conductance with dynamic clamp (Figure 1B).

a)

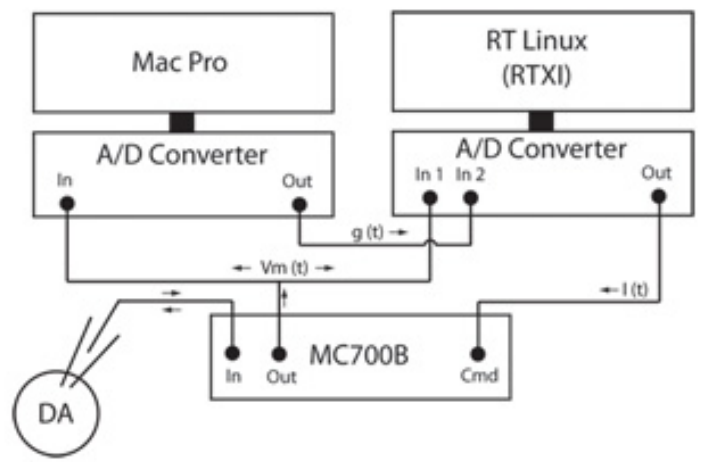

b)

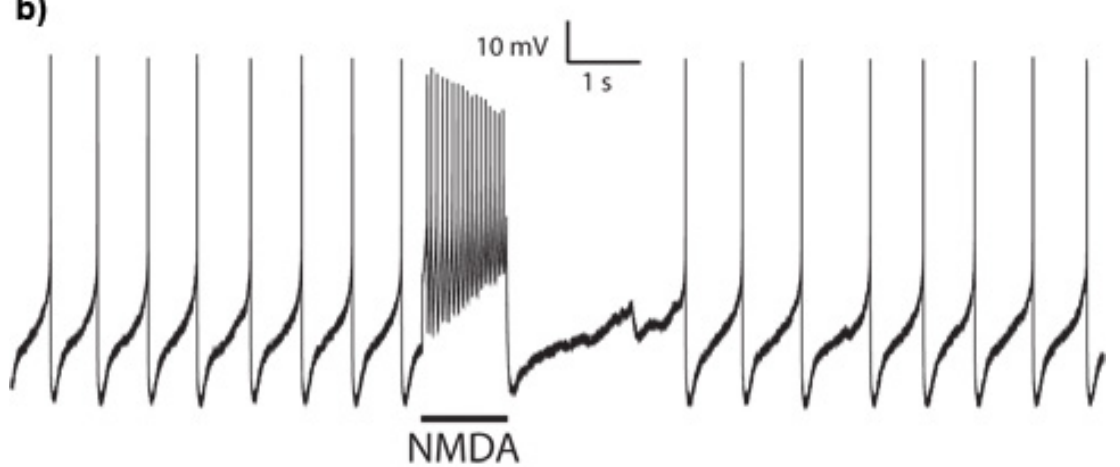

Figure 1: Application of a NMDA receptor conductance using the dynamic clamp technique. A. Hardware setup illustrating the connections between the intracellular recording rig and dynamic clamp computer. B. A burst of action potentials is evoked by application of a 40nS NMDA receptor conductance in a whole cell recording from a substantia nigra pars compacta dopaminergic neuron. 


\section{Discussion}

The dynamic clamp technique demonstrated here improves upon the traditional technique of direct current injection by allowing the experimenter to mimic the electrical effects of the activation of a receptor. In this video, we have shown that one can add the effects of activation of an NMDA receptor to the spontaneous activity of the dopaminergic neuron, i.e. a burst of action potentials are evoked.

Due to the flexibility of the hardware/software implementation, a variety of extensions can be used. The sign of the injected current can be switched from negative to positive, representing a scenario where the effects of activated receptor is removed from a neuron. Model neurons, represented in the form a series of differential equations, can also be numerically solved and allow the experimenter to investigate small networks.

\section{Disclosures}

No conflicts of interest declared.

\section{Acknowledgements}

This work was supported by MH084494 (CJL), and MH079276 and NS060658 (CAP).

\section{References}

1. Robinson, H.P., Kawai, N. Injection of digitally synthesized synaptic conductance transients to measure the integrative properties of neurons. J Neurosci Methods. 49, 157-65 (1993).

2. Sharp, A.A., O'Neil M.B., Abbott, L.F., Marder, E. The dynamic clamp: artificial conductances in biological neurons. Trends Neurosci. 16, 389-94 (1993a).

3. Sharp, A.A., O'Neil, M.B., Abbott, L.F., Marder, E. Dynamic clamp: computer-generated conductances in real neurons. J Neurophysiol. 69, 992-5 (1993b).

4. Prinz, A.A., Abbott, L.F., Marder, E. The dynamic clamp comes of age. Trends Neurosci. 27, 218-24 (2004).

5. Deister, C.A., Teagarden, M.A., Wilson, C.J., Paladini, C.A. An intrinsic neuronal oscillator underlies dopaminergic neuron bursting. J Neurosci. 29, 15888-97 (2009).

6. Lobb, C.J., Wilson, C.J., Paladini, C.A. A dynamic role for GABA receptors on the firing pattern of midbrain dopaminergic neurons. J Neurophysiol. 104, 403-13 (2010). 